\title{
AUTHOR INDEX OF VOLUME 8
}

Cockerell, Michael J., 112

Engelmann, Rudolf J., 49

Hartkopf, Günther, 74

Jastrzebski, Ludwik, 21

Kennedy, William V., 84

Kimball, Lee, 11

Kinzelbach, Wolfgang K.H., 78
Kromarek, Pascale, 57, 119

MacNeill, Jim W., 17

Rest, Alfred, 21

Schneider, Adolf R.H., 11

Train, Russell E., 128

Wright, Frank B., 116 